\title{
Translational medicine and transnational culture
}

\author{
Eugenio Pompeo \\ Department of Thoracic Surgery, Policlinico Tor Vergata University, Rome, Italy, Rome, Italy \\ Correspondence to: Prof. Eugenio Pompeo, MD, PhD, FETCS. Section of Thoracic Surgery, Dipartimento di Biomedicina e Prevenzione, Tor Vergata \\ University, Via Montpellier, 1, Rome, Italy. Email: pompeo@med.uniroma2.it.
}

Received: 19 July 2016; Accepted: 20 July 2016; Published: 05 August 2016.

doi: $10.21037 /$ vats.2016.07.01

View this article at: http://dx.doi.org/10.21037/vats.2016.07.01

Translational medicine (TM), a discipline within biomedical and public health research, is aimed at improving the health of individuals and the community by translating findings into diagnostic tools, drugs, procedures, policies and education.

Applications of TM continue to expand and the definition itself embraces different scientific areas including basic sciences such as Biology and Genetics and clinical sciences such as Surgery, Medicine and Oncology.

When dealing with research, the main aim of TM, is to translate new knowledge, mechanisms, and techniques generated by advances in basic science research into new approaches for prevention, diagnosis, and treatment of disease. This concept has been often summarized through the journalistic expression from bench to bedside. However, successful health interventions in hospitals, homes, and statehouses also require the translation of off-bench knowledge from other basic sciences including epidemiology, behavioral science, psychology, communication, cognition, social marketing, economics, and political science (1).

A further setting in which TM is called into question is the translation from pilot and phase 1 studies into broader and more extensive settings including controlled unicenter and multicenter investigations.

Several models have been proposed to distinguish the different phases and areas of influence of translational research although one of the more straightforward consider two main translational blocks. The first as the transfer of new understandings of disease mechanisms gained in the laboratory into the development of new methods for diagnosis, therapy, and prevention and their first testing in human. The second as the translation of results from clinical studies into everyday clinical practice and health decision making (1).

It is thus evident that TM can have different meanings but is becoming more and more important to almost everyone. In fact, in recent years translational research is increasingly considered a priority in many countries and a major push has been made to fund it, especially within biomedical research. In the USA since 2006 the National Institutes of Health has started forming centers of translational research and has promoted the Clinical and Translational Science Award program. In Europe, between 2007 and 2013 the European Commission devoted a majority of its $€ 6$ billion budget for health research to fund TM (1-3).

Scientific journals including Translational Medicine, the Fournal of Translational Medicine and the Annals of Translational Medicine, are specifically devoted to TMrelated publications. These journals share a common platform directed to virtually all specialties to facilitate an inter-specialty exchange of ideas, approaches and strategies, which can be selectively adopted and re-adapted by the readers according to their specific purposes.

Another important pathway which can be considered within the scopes of TM is related to the transmission of medical scientific knowledge for educational and training purposes.

In this context, when looking at a specific clinical area such as surgery we can consider both virtual and reallife educational pathways. A virtual pathway regards the translation of practical surgical knowledge through web facilities such as video sessions and video web sites and more recently even through smart-phone applications. Indeed today diffusion of videos dealing with new surgical procedures through dedicated web media, which can be easily made available to an extraordinary number of 
surgeons, has rapidly become the simplest and most widely adopted way to diffuse surgical technical knowledge. This reliable model of exchange of surgical knowledge probably represented one of the most important recent advances when dealing with didactic purposes.

On the other hand, a real-life pathway regards, apart from the participation to typical international scientific meetings, also movement of surgeons in foreign Countries and Institutions to favour an on-site direct exchange of experience, sharing of opinions and of practical surgical knowledge resulting from a face-to-face interaction developed in more restricted and confidential environments such as the operating room, hospital wards, laboratories etc.

In fact, it is particularly during these kind of encounters that that cultural and personality differences amongst the participants can more easily match each other exerting more deeply a number of effects and thus potentially affecting the individual behaviour, reasoning attitudes, and problem solving capacity. As a result maintenance of human contacts across countries to meet each other, discuss about technical and scientific issues and also enjoy the opportunity to stay together with other researchers in their natural environment being immersed in the hosts culture is another important task that in my opinion must be preserved and as yet combined with the easier opportunity to adopt web facilities.

Indeed, when dealing with surgeons the most stimulating environment certainly is the operating room, the site where every surgeon spend most of life time and where advances in surgical techniques, which have been previously analysed and realized are eventually translated into daily practise. The operating room becomes thus somewhat as a holy site in which the manual work of the surgeon can translate into the miracle of cure, the real beauty of surgery, the magic of making by borrowing a sentence pronounced by the magician Merlin in a famous movie (4). Indeed, Surgery requires perfect theoretical knowledge, continuing practise and periodic update. Not only surgeons must attend first and perform subsequently many surgical procedures during a long surgical training but must continue to up-to-date their technical knowledge to face the continuing changes characterizing both the technological and cultural evolution of surgery, learn new techniques and sometimes compare their knowledge with that of other surgeons.

During these kind of restricted encounters the daily schedule is usually quite tight and include attending surgical procedures into the operating theatre, participation to internal meetings, visits to University Institutions but also enjoyment of some convivial and relaxing opportunities. As a visiting researcher, not only a surgeon can receive useful technical insights from colleagues who perform a surgical procedure in a different way as a consequence of differences in surgical schools, health care dictates and personal preference, but can commonly learn also a cohort of small tricks originating from the experience and inventive of the surgeon, who often refines in a personalized way the various techniques according to his/her individual surgical skills and attitudes. Moreover, the opportunity to live in a foreign Country in the working environment of other colleagues can offer the opportunity to add to an exchange of scientific knowledge, an absorption of cultural insights from the hosts and hopefully a release of insights belonging to the visitor native culture (1).

This transnational culture process calls into question a number of factors including an open-minded approach and as yet the customs, lifestyle and other cultural aspects of each individual who is actively involved in the process and I would say that the more diverse are the cultural differences amongst the participants the most stimulating the net result of this matching.

In fact, when generally speaking, the concept of transnational culture is strictly dependent by another multifaceted and as yet relatively well investigated process related to the flows of people moving from one Country to another denominated transnationalism. In the globalization era transnationalism's central interest is circulation, movement, and exchange of things, data, images and concepts. As a result transnational flux of culture can practically offer a series of innovative options deriving from a culturally different environment, which incidentally or deliberately matches with a hosting environment. It is highly probable that we all have already experienced some effects of transnationalism since most of the large cities worldwide are now widely multiethnic and are affected at least in some social aspects by such a process including sharing of arts, politics, sports, eating habits etc.

When dealing with research-related environments, the transcultural process can thus lead to a continuous deterritorialized injection of new meaning streams into the group in agreement with a real globalization of culture, which must be clearly distinguished however by its homogenization. More specifically, when the encounter is motivated by a common professional objective or interest the result can be an extraordinary and enriching event, which apart from a stimulating exchange of technical knowledge may also lead to more subtle and as yet less 


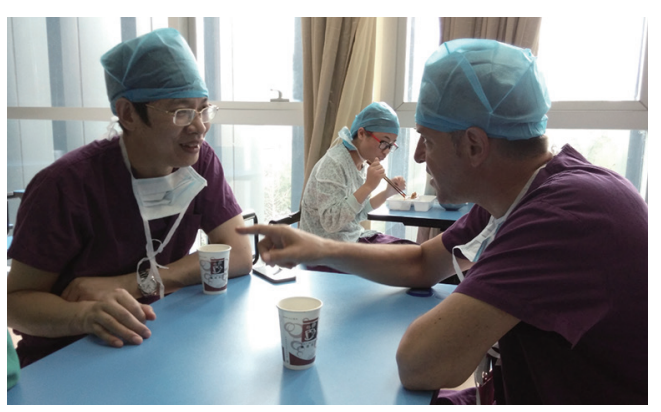

Figure 1 An active discussion between Dr. Qixun Chen (left) and me (right) during a coffee break at the Zhejiang Cancer Hospital in Hangzhou.

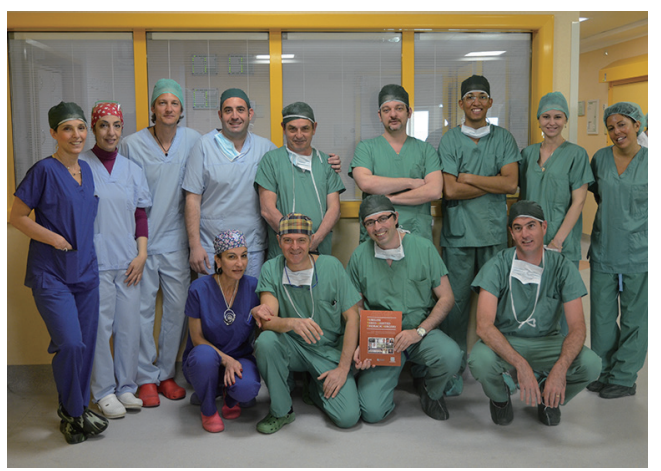

Figure 2 A typical group picture in the operating room of the Tor Vergata University Hospital showing hosts and visiting Professors (within the first line Dr M. Papiashvili from Israel is the second from the right; I am the third).

immediately perceived exchange of cultural insights thus defining a more complex effect that I would arbitrarily define a transnational culture effect (TCE) and that is in anyway facilitated by the recognition of symbolic common denominators that help interact the group members irrespective of any difference in language, race and culture (5) (Figure 1).

It is conceivable that as a consequence of the TCE the interaction of people can thus lead to a complex exchange if inputs including a wide range of insights that are released during these multifaceted interactions by people with different behavioural, social and cultural habits. These insights, might be subsequently stored in variable degree by the group participants eventually enriching the overall individual cultural baggage and sometimes affecting in a meaningful and as yet durable way not only surgical practise but even other aspects of the way of thinking and lifestyle.
These types of inter-Institutional movements of researchers is clearly not a new representing a consolidated practise worldwide (Figure 2). Nonetheless in recent years China, is assuming a leading role in this regard. In fact, on one hand, the enormous amount of surgical procedures, which are routinely daily performed in some university hospitals in China is rendering these Institutions highly attractive for surgeons coming from western Countries in which such a patients turnover would be not even imaginable. On the other hand, China is actively promoting the sharing and exchange of experience between Chinese and foreign surgeons. In this way both parties can mutually take advantages. Non-Chinese surgeons have the opportunity to attend in a relatively short time period a huge number of surgical procedures related to particular types of surgical approaches and specific disease treatments, which can be concentrated within relatively short time periods (6). On the other, Chinese surgeons can implement their knowledge with different research activity models, which are employed in other Countries, sharing their own protocols and creating networks and new opportunities to cooperate at an international multi-institutional level.

During a flight back to Italy at the completion of a 10 days trip including a visit to several University Institutions from the north to the south of China, the video placed in front of me informed that the flight distance between Rome and Guangzhou was of about 9,500 km. When making a mental summary of my recent experience, I satisfactorily realized that I received useful surgical knowledge insights and hopefully contributed to release as a counter-effect few inputs to the hosts. At the same time I realized to have benefited as well by a TCE that has affected in a less immediately perceived manner my personal attitudes and way of thinking and probably of making. Indeed, the great interest I felt while attending quite many surgical procedures in prestigious Chinese Institutions was blended in my mind with the vivid vision of the proud terracotta warriors in $\mathrm{Xi}$-an, a bike riding along the West Lake in Hangzhou, or a relaxing visit to an old tea house in Chengdu.

Surprisingly enough I was reasoning about these issues while tasting a delicious Chinese dinner using the chopsticks quite as comfortably as forks and knife.

\section{Acknowledgements}

I thank the AME Company that offered me the opportunity to accomplish my visits in China. I also would like to 
express my deep gratitude and sincere estimation to Ms. Melanie He who nicely and efficiently accompanied me during a 10-days trip across China.

\section{Footnote}

Conflicts of Interest: The author has no conflicts of interest to declare.

\section{References}

1. Woolf SH. The meaning of translational research and why it matters. JAMA 2008;299:211-3.

2. Trochim W, Kane C, Graham MJ, et al. Evaluating

doi: 10.21037/vats.2016.07.01

Cite this article as: Pompeo E. Translational medicine and transnational culture. Video-assist Thorac Surg 2016;1:3 translational research: a process marker model. Clin Transl Sci 2011;4:153-62.

3. Sung NS, Crowley WF Jr, Genel M, et al. Central challenges facing the national clinical research enterprise. JAMA 2003;289:1278-87.

4. Boorman J. Excalibur (movie). Orion Pictures Corporation (USA, UK). 1981.

5. Willis DB. Transnational culture and the role of language: an international school and its community. Peen State Eds. 1992;41:73-95.

6. Sihoe AD. Opportunities and challenges for thoracic surgery collaborations in China: a commentary. J Thorac Dis 2016;8:S414-26. 